\title{
The Decision of Garment Return Service Based on Supply Chain with All-Channel
}

\author{
Mei-zhi Chen ${ }^{1}$, Jia-li Pan $^{1}$ and Xing-jian Zhou ${ }^{1,2, *}$ \\ ${ }^{1}$ School of Management, Wuhan Textile University, Wuhan, China \\ ${ }^{2}$ Research Center of Enterprise Decision Support, Hubei Province Research Base for Humanities and Social Sciences, \\ Wuhan, China \\ Email: wuliuwtu@163.com
}

\begin{abstract}
All-channel operation is to improve the customer's purchase experience as the core, not only requires each channel to maintain its own high standards of service, at the same time to achieve inter-channel services synergies, which undoubtedly put forward higher requirements for the enterprise's supply chain management. In the paper, aiming at the competitive advantage of allchannel service providers, we determine that all-channel supply chain can satisfy the future market's products/services, and transform the product/service requirements into tasks and assets of processes and various sub-processes through big data analysis. According to the market demand, we coordinate the supply chain and achieve continuous evaluation. Applying Bayesian network and reasoning network graph model, the decision-making of garment supply chain all-channel return service is carried out through four steps: service demand forecast, product/service demand segmentation, data analysis, data interpretation and decision-making. The simulation results show that the optimal expected profit can be obtained by optimizing the combination of return services through capacity set network graph for different return services in online and offline channels.
\end{abstract}

Keywords: all-channel; garment supply chain; return service decision

\section{全渠道供应链下服装品退货决策及仿真分析}

\author{
陈美芝 ${ }^{1}$, 泮家丽 ${ }^{2}$, 周兴建 ${ }^{1,} 2^{*}$ \\ 1 武汉纺织大学 管理学院, 武汉 \\ 2 湖北省人文社会科学研究基地 企业决策支持研究中心, 武汉 \\ Email: wuliuwtu@163.com
}

\begin{abstract}
摘要: 允许退货日益成为提升企业利润的一项竞争战略, 全渠道运营以提高顾客的购买体验为核心, 不仅要求各渠道保持自身高服务水准, 同时要实现渠道间服务的相互协同, 这无疑给供应链运营管 理上提出了更高的要求。本文以获得竞争优势为主旨, 确定服装全渠道供应链可以满足未来市场的 产品/服务, 结合大数据分析将产品/服务需求转化为流程和各类子过程的任务和资产, 根据市场需 求, 通过供应链协调并达到持续评估。应用贝叶斯网络和推理网络图模型, 通过服装品退货服务需 求预测、细分产品/服务需求、数据分析、数据解释和决策等四个步骤进行服装供应链全渠道退货服 务决策。仿真分析表明, 线上渠道和线下渠道针对不同的退货服务, 通过能力集网络图优化寻找最 佳退货服务组合，可获得最优期望利润。
\end{abstract}

关键词：全渠道，服装供应链，退货决策

\section{1 引言}

随着网络购物市场规模的扩大，更多的企业加入了在线零售市场，购物手段日趋多样化 ${ }^{[1]}$ 。一般来说， 价格敏感相对年轻、购买相对方便、比较时尚的顾客偏好于电子渠道, 而年长者并享受这街乐趣的顾客 
偏好于传统渠道（Schroder 等, 2008） ${ }^{[2]}$ 。但为了提高消费者的满意度、忠诚度和在线零售商的竞争力, 允许退货日益成为提升企业利润的一项竞争战略（Frankel R 等, 2011) ${ }^{[3]}$ 。对退货服务的重视的确帮 助企业吸引顾客和刺激消费上起到了较大作用, 虽然退货现象也给企业带来了逆向物流处理成本的有关 问题 ${ }^{[4]}$, 然而在激烈竞争的市场环境中, 退货决策及管理确实创造了更好的客户价值（Ai X 等，2012） ${ }^{[5]}$ 。

现有对退货决策的研究, 主要有三个方面: (1)将 “退货” 作为一个前置条件进行相关研究, 多针对 线上电子渠道, 如, 翟春娟等 (2011) ${ }^{[6]}$ 研究不同的退货策略下, 在线零售商以及整个供应链所得利润 差异; 孙军等 (2014) ${ }^{[7]}$ 研究了无缺陷退货条件下的在线零售商在销售之初选择权变策略、免运费和分 离运费三种运费承担策略对利润、消费者购买决策、消费者需求量和退货量的影响; (2)在一定前置条件 下研究 “退货” 问题, 多针对线下传统渠道, 如, 李勇建等 (2012) ${ }^{[8]}$ 研究了在产品需求和消费者产品 估价均不确定的情况下, 报童零售商的预售策略和无缺陷退货问题; 杨光勇等 (2014） ${ }^{[9]}$ 研究了存在战 略顾客时, 不再销售、正常再销售与降价再销售退货产品策略如何影响销售商的顾客退货策略设计; 张 福利等 (2017) ${ }^{[10]}$ 基于理性预期和战略顾客行为, 研究不确定需求条件下零售商的四种退货策略; 何啸 源等 (2018) ${ }^{[11]}$ 研究在线零售商的最优退货策略, 以及部分退款退货选项对在线零售商决策的影响; (3) 供应链上的 “退货” 问题, 分别针对线上或线下不同的渠道, 如, 姚忠 (2008) ${ }^{[12]}$ 分析了在零售商在风 险约束下，退货策略对单周期供应链的协调性问题; 汪峻萍等（2013） ${ }^{[13]}$ 针对网上在线销售易逝品的无 缺陷退货现象, 研究供应链的最优订购协调问题; 李新军等 (2007) ${ }^{[14]}$ 研究了随机需求条件下基于退货 的单周期单一产品逆向供应链模型; 杨鹏等 (2007) ${ }^{[15]}$ 针对供应链中无质量缺陷的退货问题, 建立了单 个制造商和零售商构成的分散决策模型, 研究零售商宣传咨询对于顾客需求和退货率的影响, 等等。

但是，在新零售时代的全渠道背景下，退货决策与管理如何在线上线下各个供应链渠道间协作和互 补成为新的问题, 此前文献也较少涉及, 对于服装品这一受到退货策略重要影响的领域 ${ }^{[16]}$, 更为缺乏相 应的研究。本文正是在这一背景下, 考虑全渠道供应链情景, 研究服装品退货服务决策机制及其供应链 协作模式。

\section{2 供应链全渠道服务决策问题描述}

服装品一般具有季节性、时效性、流行性等的特点, 在七天无理由退货服务政策下, 企业不断提高自身 渠道的资源配置和相应的能力建设, 以期实现整合各渠道优势, 满足客户更高的购物需求, 形成良性的 互动和服务 (周飞等, 2017) ${ }^{[17]}$ 。

因此, 在供应链全渠道下, 以获得竞争优势为主旨, 服装生产/销售企业应用大数据分析将产品/服 务需求转化为流程和各类子过程的任务和资产, 根据市场需求进行供应链协调。服装品供应链全渠道服 务决策问题可用如下过程描述:

（1）通过大数据分析识别产品/服务创新面临的市场需求;

(2) 将产品/服务需求细分到研发、生产、销售、售后等各个过程;

（3）针对每个过程分别进行分析优化;

（4）遵照产品/服务持续创新, 满足市场需求, 实现全渠道供应链协调。

这是一个不断循环、不断改进的过程。具体如下图 1 所示。

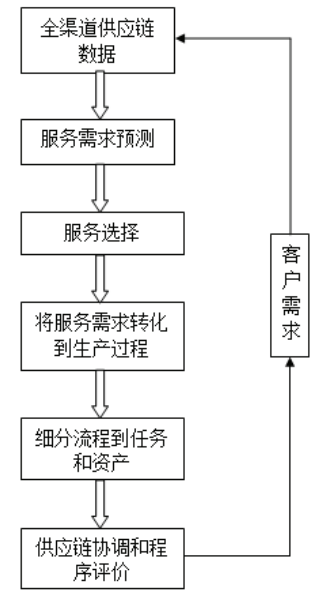

图 1. 供应链全渠道服务决策框架 


\section{3 服装供应链全渠道退货服务决策模型}

\section{1 供应链退货服务需求预测}

在实际应用中，供应链服务需求是受多方影响的。如，以淘宝、天猫发起的服装品 7 天无理由退货政策， 对供应链退货服务需求的增长产生了极大影响。考虑到服装品的特点, 根据用户消费习惯等大数据, 可 通过贝叶斯网络对数据进行预测和精炼。具体流程如下:

(1) 收集数据。预测的基础通常来源于购买行为、搜索记录以及社交评价, 毫无疑问数据会对消费 者偏好造成显著影响。从服装品全渠道供应链数据中选取合适的样本集, 服装品制造商/零售商结合服 装行业先验经验、服装品销售平台买家评价或参考专家建议, 通过数据挖掘、大数据技术等手段, 识别 影响供应链退货服务需求的因素。一旦这些因素被识别, 将作为贝叶斯网络上的节点 $X_{i}(i=1$, $2 ， \cdots, n)$ 。服装品制造商/零售商须收集多个具有代表性的消费者，对每个节点进行赋值。 法建模。

(2) 预处理样本数据。运用聚类分析或者分层分类等方法确保各因素赋值不连续, 便于后续推理算

(3) 设计和建立贝叶斯网络。贝叶斯网络由代表变量节点及连接这些节点的有向边构成, 一个节点 代表随机变量, 节点间的有向边代表了节点间的互相关系, 用条件概率进行表达关系强度, 没有父节点 的用先验概率表达信息之间的关系 (藏玉卫等, 2003) ${ }^{[18]}$ 。每个节点 X 都有一个概率分布 $P(X / \pi$

$(X))$, 表示相关变量的不确定性, 其中 $\pi(X)$ 代表父节点 $X$ (如果节点 $X$ 中的 $\pi(X)=\varnothing$ 代表非父节 点)。因此在独立性假设下, 一个 $n$ 节点构成的贝叶斯网络 $\left(X_{1}, X_{2}, \cdots, X_{n}\right)$ 可以表述为联合概率分布:

$$
P\left(X_{1}, X_{2}, \cdots, X_{n}\right)=P\left(X_{i} / \pi\left(X_{i}\right)\right)
$$

(4) 预测服务需求。由于知晓各个节点的赋值, 服装品制造商/零售商可以通过计算目标节点的概率 分布来预测供应链退货服务需求。

(5) 敏感性分析。 $M I$ (交互信息) 可以衡量两个随机变量间的依赖关系, 适合贝叶斯网络的敏感性 分析。如通过了解变量 $A$ 可以减少变量 $B$ 的不确定性, 反之亦然。变量 $A$ 与 $B$ 的 $M I$ 表述为:

$$
T(A, B)=\sum_{a, b} P(A, B) \log \frac{P(A, B)}{P(A) P(B)}
$$

其中, $P(A, B)$ 表示联合概率分布, $P(A)$ 表示 $A$ 的概率分布函数, $P(B)$ 表示 $B$ 的概率分布函 数; $T(A, B)$ 表示变量 $A$ 和 $B$ 间相互影响关系, 其值越大说明变量 $A$ 和 $B$ 间关系越紧密, 故可根据 $T(A$, $B)$ 的大小来判断变量的重要性。在实际中, $T(A, B)$ 越大, 越需要关注相关变量。此外, 贝叶斯网络可 以及时反映变化的市场需求, 服装品制造商/零售商可以通过持续改进贝叶斯网络来驱动供应链退货服 务的持续创新。

\section{2 细分供应链退货服务需求}

经过第一步供应链退货服务需求预测后, 借助于推理网络图模型, 允许企业把自己的能力集和其他企业 的合并, 通过链接来自不同纺织服装企业的资源能力集, 将供应链退货服务需求细分到产品设计、生产、 销售、售后等各个过程，可采用可视化的方式提供一系列最优的扩展细分过程。

\section{3 供应链退货服务数据分析}

使用推理网络图模型能够说明供应链退货服务能力集的扩张过程, 这是与其他能力合作的一种网络优化 模型。假定 $E$ 是供应链退货服务需要解决的问题, $T R$ 是真正需要的能力集, $S K$ 是可获得解决具体问题 的能力集, 中介技能 $(I)$ 可以提高学习速度或连接 $T R$ 和 $S K$ 模型, 以帮助决策者从 $S K$ 获 $T R$ 。该方法的 基础是试图从起始节点建立一个推理网络图 $(S K)$ ，从开始到中间节点 $(I)$ 再到结束节点 $(R)$, 然后利用 0-1 整数规划寻求最优解。因内部能力 (I)、现有能力集 (SK)、所需能力集 (TR) 和相关技能的习惯领域 以及学习成数据输入到推理图, 可以构建服装品全渠道供应链退货服务行为演化机理的一个特定的数学 模型（如网络流优化模型）。

\section{4 供应链退货服务决策}

利用推理网络图模型进行数据分析, 提供一个基于学习网络连接相关的能力集合, 然后利用优化程序来 帮助全渠道供应链退货服务决策者获取所需技能的最佳解决方案, 同时提供解决问题的更多替代过程序 列。以此为基础, 将网络关系拓展到整个全渠道供应链服务流程中, 做出最佳的决策。将供应链退货服 
务需求进行细分, 帮助服装品制造商/零售商建立具体的分析流程, 以协调全渠道供应链, 驱动供应链 退货服务持续创新或改进。

\section{4 仿真分析}

\section{1 参数设计}

设某服装销售企业精选出了 5 种不同类型的渠道服务 $A 、 B 、 C 、 D 、 E$ （如退货限期、退货补偿、上门 退货服务等），并识别出各个退货服务需要的能力集（如表 1 所示）。表中 $a 、 b 、 c 、 d 、 e 、 f 、 g 、 h 、$ $i 、 j 、 k 、 1 、 m 、 n$ 表示不同的能力集。同时，进行如下假定:

(1) 不同的退货服务需要不同的能力集。例如, 退货服务 $A$ 需要能力集 $g 、 f$, 而退货服务 $B$ 需要 能力集 $g 、 f 、 i$ 。

(2) 有线上和线下两个渠道，线上渠道拥有退货服务能力集 $c 、 d 、 e$; 线下渠道拥有退货服务能力 集 $a 、 b 、 f_{0}$ 线上渠道和线下渠道都不能单独为退货服务 $A 、 B 、 C 、 D 、 E$ 提供所需的能力集。这时为了 提供相应的退货服务, 每个渠道需从其他渠道购买, 或通过学习现有的能力集来获取新的能力集。假设 能力集的销售价格如表 2 所示。其中, 能力集 $c$ 的销售价格为 1 单位, 能力集 $f$ 的销售价格为 1.5 单 位。

表 1. 各退货服务需要的能力集

\begin{tabular}{|c|c|c|c|c|c|c|c|c|c|c|c|c|c|c|}
\hline & $a$ & $b$ & $c$ & $d$ & $e$ & $f$ & $g$ & $h$ & $i$ & $j$ & $k$ & 1 & $m$ & $n$ \\
\hline$A$ & & & & & & $\sqrt{ }$ & $\sqrt{ }$ & & & & & & & \\
\hline$B$ & & & & & & $\sqrt{ }$ & $\sqrt{ }$ & & $\sqrt{ }$ & & & & & \\
\hline$C$ & & & & $\sqrt{ }$ & & & & $\sqrt{ }$ & & & & & $\sqrt{ }$ & $\sqrt{ }$ \\
\hline$D$ & & $\sqrt{ }$ & $\sqrt{ }$ & & & & & & & $\sqrt{ }$ & & & & \\
\hline$E$ & & & & & & $\sqrt{ }$ & & & & & $\sqrt{ }$ & $\sqrt{ }$ & & \\
\hline
\end{tabular}

表 2. 能力集的销售价格

\begin{tabular}{lcc}
\hline & $c$ & $f$ \\
\hline 线上渠道 & 1 & \\
线下渠道 & & 1.5 \\
\hline
\end{tabular}

（3）线上渠道退货服务能力集的成本如表 3 所示, 线下渠道退货服务能力集的成本如表 4 所示。需 要注意的是，实际操作中购买/学习能力集的成本需考虑到时间、劳动力、资源、资金等各个方面因素。

表 3. 线上渠道扩展/学习能力集的成本

\begin{tabular}{rrrrrrr}
\hline & $f$ & $g$ & $h$ & $i$ & $m$ & $n$ \\
\hline$c$ & 2 & 1.5 & & 1 & & \\
$d$ & & & 2 & 1.5 & & \\
$e$ & 2.5 & & & & 1 & \\
$f$ & & 1 & & & & 2 \\
$g$ & & & & 1.5 & & \\
$h$ & & & & & & 2.5 \\
$i$ & & 1 & & & & \\
$m$ & & & & & & \\
$n$ & 1 & & 1.8 & & & \\
$d^{n} e$ & 1 & & 1 & & & 1.5 \\
\hline
\end{tabular}


表 4. 线下渠道扩展/学习能力集的成本

\begin{tabular}{|c|c|c|c|c|}
\hline & $c$ & $j$ & $k$ & 1 \\
\hline a & 1.8 & & 2 & \\
\hline$b$ & & & 1.8 & \\
\hline$c$ & & 0.8 & & \\
\hline$f$ & 1.5 & 1 & & \\
\hline$j$ & 1 & & & \\
\hline k & & & & 1 \\
\hline 1 & & 1.5 & & \\
\hline$a \hat{b}$ & 1 & & 1.5 & \\
\hline
\end{tabular}

\section{2 决策过程}

考虑到供应链退货服务能力集的学习/购买成本，线上渠道应该专注于退货服务 $A 、 B 、 C$; 而线下渠道 应该专注于退货服务 D、E。假设线上渠道和线下渠道各自只能提供一种退货服务, 则此时将存在 6 种 退货服务组合，各退货服务组合的期望收益如表 5 所示。

表 5. 退货服务组合的期望收益

\begin{tabular}{ccc}
\hline 退货服务组合 & 线上渠道 & 线下渠道 \\
\hline$A, D$ & 8 & 4.5 \\
$A, E$ & 7 & 5 \\
$B, D$ & 5.5 & 4 \\
$B, E$ & 6 & 3.5 \\
$C, D$ & 5 & 3 \\
$C, E$ & 4 & 3.5 \\
\hline
\end{tabular}

分别构建线上渠道和线下渠道的退货服务能力集网络图, 如图 2 和图 3 所示。其中每个节点示一个 能力集, 边表示能力集间的关系。例如, $e \rightarrow f$ 表示能力集 $\mathrm{f}$ 可以通过学习能力集 $e$ 来获取, 而节点 $m$ 和 $g$ 之间没有边则表示能力集 $\mathrm{m}$ 不可能通过学习能力集 $g$ 来获取, 反之亦然。边上的数值表明获取新 能力集所需的购买/学习成本。另外, 网络图中还存在复合节点, 如 $d^{\wedge} e$ 和 $a \hat{}{ }^{\wedge} b$, 即复合能力集。为了 提供相应的退货服务均需要获取其他能力, 如线上渠道的退货服务可从线下渠道购买, 或通过学习现有 的退货服务能力集来获取新的能力集。事实上, 对能力集 $\mathrm{c}$, 线下渠道可以通过学习能力集 $f 、 a b 、 a$ 来获取, 也可以向线上渠道来购买。获取的成本有高有低, 分别为 $1.5 、 1 、 1.8$ 和 1 。综上, 构建上述 退货服务能力集网络图的最终目标是使用优化方法找出最佳的全渠道供应链退货服务组合, 实现供应链 利润最大化。

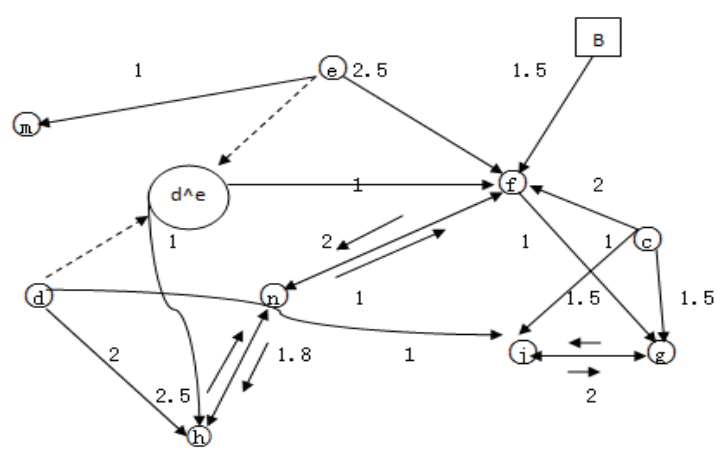

图 2. 线上渠道退货服务能力集网络

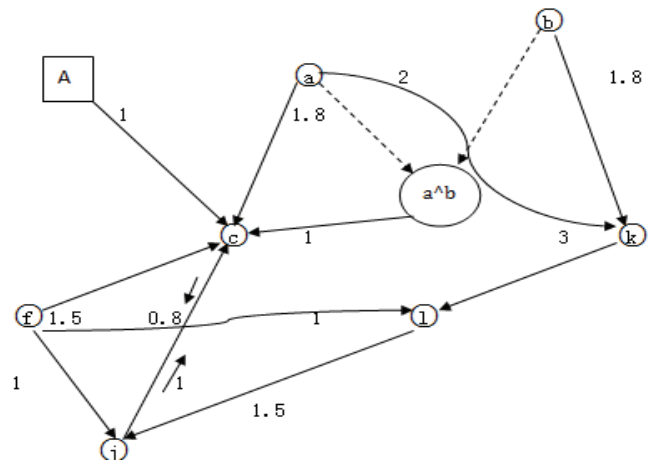

图 3. 线下渠道退货服务能力集网络

\section{3 模型求解}

运用网络流的方法来寻求最优解。进行如下假设： 
假设 1：各渠道需列出所有相关的信息，例如，各退货服务能力集和相关成本。

假设 2: 各渠道须相互合作。

假设 3: 单一渠道只需支付一定的价格, 就可以从其他渠道自由地购买所需的退货服务能力集。

假设 4: 各渠道与该服装销售企业是利益共同体。

用 $S$ 表示渠道现有的退货服务能力集, $T$ 表示提供退货服务需要的能力集, $I$ 表示媒介(中间)能力 集。定义一个有向图 $G=(V, E), V=T \cup I \cup S$, 假设节 $i$ 在有向图 $G=(V, E)$ 上, $r(i, j)$ 表 示节点 $i$ 接节点 $j$ 的边; $z(i, j)$ 表示通过节点 $i$ 获取节点 $j$ 的成本。设网络图初始节点 $S_{0}$, 终端节 点为 $t_{0}$ 。连接 $S_{0}(S \in S)$ 和 $t_{0}(t \in T)$, 得到新的有向图 $G=\left(V^{\prime}, E^{\prime}\right), n$ 表示 $T$ 的价值。得到容量和费 用的边界如下:

$$
\begin{aligned}
& c(i, j)=\left\{\begin{array}{l}
1, r(i, j)=r\left(t, t_{0}\right) \\
\mathrm{n}, r(i, j) \neq r\left(t, t_{0}\right)
\end{array}\right. \\
& z(i, j)= \begin{cases}0, r(i, j)=r\left(s, s_{0}\right) & \text { 或 } r(i, j)=r\left(t, t_{0}\right) \\
z(i, j), r(i, j) \neq r\left(s_{0}, s\right) \text { 且 } r(i, j) \neq r\left(t, t_{0}\right)\end{cases}
\end{aligned}
$$

用 $f(i, j)$ 表示边 $r(i, j)$ 的流量, 得到供应链退货服务最小费用流模型如下:

$$
\operatorname{Min} \sum_{(i, j) \in E^{\prime}} z(i, j) f(i, j)
$$

s.t

$$
\sum_{x} f(i, x)-\sum_{x} f(x, i)=\left\{\begin{array}{l}
n, \quad i=S_{0} \\
-n, \quad i=t_{0} \\
0, \quad i \neq S_{0} \text { 且 } i \neq t_{0}
\end{array}\right.
$$

$0 \leq f(i, j) \leq c(i, j)$

\section{4 结果分析}

运用软件 $L I N G O$ 可得到模型的最优解 (如表 6 所示), 并得到线上渠道和线下渠道退货服务最优解对 应的推理网络图 (图 4、图 5 所示) 。结果表明, 全渠道供应链最优退货服务组合 $\{\mathrm{A}, \mathrm{D}\}$, 获得 8.2 单 位的期望利润。

表 6. 仿真实验结果

\begin{tabular}{ccc}
\hline & 线上渠道 & 线下渠道 \\
\hline 选择的退货服务 & $\mathrm{A}$ & $\mathrm{D}$ \\
需要的能力集 & $\{\mathrm{f}, \mathrm{g}\}$ & $\{\mathrm{j}, \mathrm{c}, \mathrm{b}\}$ \\
学习获得的能力集 & $\{\mathrm{g}\}$ & $\{\mathrm{j}\}$ \\
购买获得的能力集 & $\{\mathrm{f}\}$ & $\{\mathrm{c}\}$ \\
总成本 & 2.5 & 1.8 \\
收益 & 8 & 4.5 \\
\hline
\end{tabular}

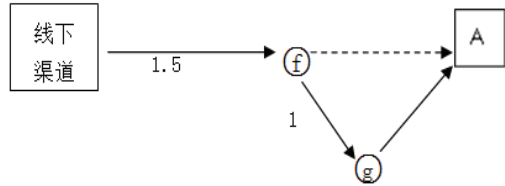

图 4. 线上渠道的最优解对应推理网络图

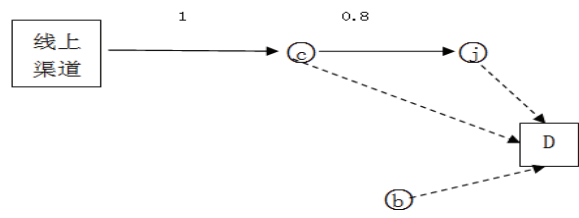

图 5. 线下渠道的最优解对应推理网络图

\section{5 结束语}

供应链全渠道服务决策问题可描述为通过大数据分析识别产品/服务创新面临的市场需求, 进而将产品/ 服务需求细分到研发、生产、销售、售后等各个过程, 然后针对每个过程分别进行分析优化, 遵照产品 
/服务持续创新, 实现全渠道供应链协调。应用贝叶斯网络和推理网络图模型, 通过对某服装销售企业 5 种不同类型的渠道服务及相应退货服务决策的仿真分析, 结果表明: 服装企业利用线上渠道和线下渠 道针对不同的供应链退货服务进行退货服务决策，企业运营管理者可利用线上线下全渠道退货服务能力 集网络图寻，找最佳的全渠道供应链退货服务组合，实现供应链利润最大化。

致谢. 本文得到 2019 年中国物流学会、中国物流与采购联合会面上研究课题计划、2019 年湖北省教育 厅人文社科项目和武汉纺织大学 2018 年科技创新计划的资助, 是其阶段性成果。

\section{参考文献}

1. 张涛,李刚,罗美玲,黄冯风.考虑无缺陷退货的在线商品信息发布策略[J]. 运筹与管理, 2017,26(3) :123-130.

2. Schroder H, Zaharia S.. Linking multichannel customer behavior with shopping motives: an empirical investigation of a German retailer [J]. Journal of Retailing and Consumer Services,2008,15(6) : 452-468.

3. Mollenkopf D A, Frankel R, Russo I.. Creating value through returns management : Exploring the marketing operations interface [J]. Journal of Operations Management. 2016,29(5) : 391-403.

4. 李冉. 服装企业退货管理的逆向物流分析[J].物流科技, 2011,(9):86-88.

5. Ai X., Chen J, Zhao H, Tang X.. Competition among supply chains : Implications of full returns policy [J]. International Journal of Production Economic, 2012,139(1) : 257-265.

6. 翟春娟,李勇建.B2C 模式下的在线零售商退货策略研究[J].管理工程学报,2011,25(01):62-68.

7. 孙军,孙亮.基于无缺陷退货的在线零售商运费承担策略研究 [J].软科学,2014,28(06):41-45.

8. 李勇建,许磊,杨晓丽. 产品预售、退货策略和消费者无缺陷退货行为 [J]. 南开管理评论,2012,15(05):105-113.

9. 杨光勇,计国君.存在战略顾客的退货策略研究 [J]. 管理科学学报, $2014,17(08): 23-33+94$.

10. 张福利,张燕,徐小林.基于战略顾客行为的零售商退货策略研究 [J].管理科学学报,2017,20(11):100-113.

11. 何啸源,甄学平,刘斌, 考虑双退货期限的在线零售商退货策略研究 [J].上海管理科学, 2018,40(04):85-88.

12. 姚忠.风险约束下退货合同对供应链的协调性分析[J].管理科学学报,2008,11(03):96-105.

13. 汪峻萍,杨剑波,贾兆丽.基于无缺陷退货的网上销售易逝品供应链协调模型 $[J]$.中国管理科学,2013,21(06):47-56.

14. 李新军,达庆利.随机需求条件下产品退货政策协调与优化[J].中国管理科学,2007(03):56-60.

15. 杨鹏,陈秋双,孙俊清.无缺陷退货问题的建模与供应链协作 [J].计算机集成制造系统, 2007(06):1071-1075.

16. 李泽聚,祝驿楠. 纺织服装业逆向物流联盟模式及其云计算平台研究 [J]. 经营与管理, 2016,(12):104-107.

17. 周飞,郑洁仪,沙振权.基于渠道协同视角的企业多渠道管理研究综述 [J].华侨大学学报, 2017,(5):51-62.

18. 藏玉卫,王萍,吴育华.贝叶斯网络在股指期货风险预警中的应用[J].科学学与科学技术管理, 2013(1): 122-125. 